\author{
B. BACROIX, O. BRUN, AND TH. CHAUVEAU \\ LPMTM - CNRS - Université Paris-Nord \\ Av. J.B. Clément. 93430 Villetaneuse - FRANCE
}

\begin{abstract}
Textures measured on $2 \mathrm{Al}$ alloys after hot and cold rolling are analyzed in detail. It is shown that the texture strength and the intensity of the brass component increase with increasing temperature. This is in good agreement with some texture predictions.
\end{abstract}

\title{
INTRODUCTION
}

Whereas cold deformation and recrystallization textures have been extensively studied 1,2 , little attention has been devoted to the effect of temperature on rolling texture development in the absence of recrystallization. This effect is usely considered to be negligible 3,4 . In contrast, during torsion, quite different textures have been measured in aluminium deformed at various temperatures ${ }^{5}$. In order to shed some light on this apparent contradiction, some textures are reported here, which have been measured after cold and hot rolling of $2 \mathrm{Al}$ alloys, starting from the same initial texture. After a brief description of the experimental materials and procedure, the textures measured on samples extracted from the mid-thickness of the sheets are presented. Some comparisons are made between experimental and calculated textures.

\section{EXPERIMENTAL METHODS}

Two alloys 1200 and 8979 (table 1), were first rolled in a reversible rolling mill (at Pechiney) up to a total reduction of 95\%. The finishing temperature was about $450^{\circ} \mathrm{C}$. Some samples were quenched at this stage for texture measurement and subsequent rolling. An additional reduction of $83 \%$ was then applied to the slabs on the industrial site at a temperature ranging from 420 to $265^{\circ} \mathrm{C}$ for the 1200 alloy and from 440 to $330^{\circ} \mathrm{C}$ for the 8979 material in a multipass rolling schedule. Some samples were also cold rolled in a laboratory mill up to the same final reduction.

Table 1 : Composition of alloys used, weight percent.

\begin{tabular}{|c|c|r|r|r|r|c|}
\hline Alloy & $\mathrm{Fe}$ & $\mathrm{Si}$ & $\mathrm{Zn}$ & $\mathrm{Mn}$ & $\mathrm{Ti}$ & $\mathrm{Al}$ \\
\hline 1200 & 0.57 & 0.15 & $90 \mathrm{ppm}$ & $40 \mathrm{ppm}$ & $50 \mathrm{ppm}$ & bal \\
\hline 8979 & 1.37 & 0.07 & 0.02 & $84 \mathrm{ppm}$ & $<0.02$ & bal \\
\hline
\end{tabular}


Optical micrographs of the initial materials revealed that the $1200 \mathrm{Al}$ alloy was fully recrystallized after reversible rolling (with an average grain size equal to $200 \mu \mathrm{m}$ ) whereas the 8979 alloy still presented elongated grains in most of the volume of the sheet (Figure 1). During cold and hot rolling, small sub-grains (with an average size equal to $30 \mu \mathrm{m}$ ) appear within the original coarse grains. Orientation Distribution Functions (ODF's) were calculated using both the vector ${ }^{6}$ and harmonic methods 7 .

(a)

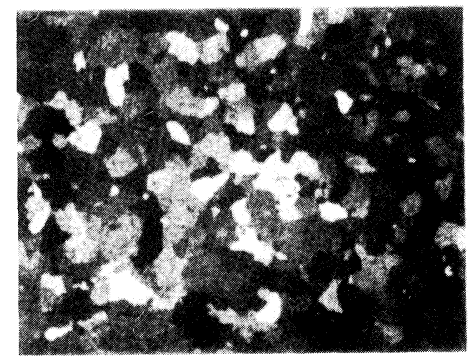

(c)

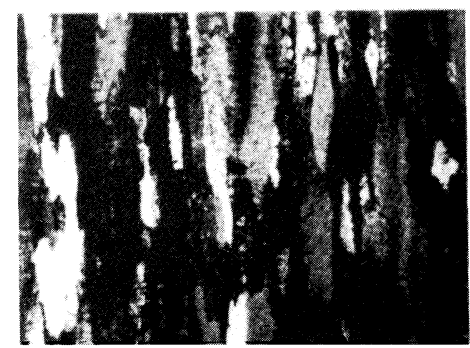

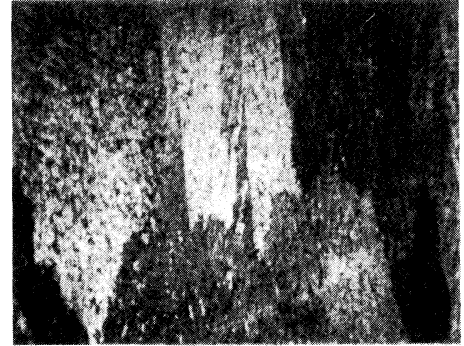

(b)

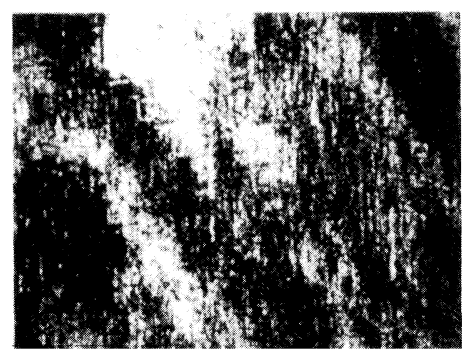

(d)

Figure 1 Microstructures of the 2 alloys after reversible (RR) and hot (HR) rolling : a) 1200-RR, b) 8979 - RR, c) $1200 \mathrm{HR}$ and d) $8979 \mathrm{HR}$ (magnitude x 14).

\section{EXPERIMENTAL TEXTURES}

The recalculated $\{111\}$ pole figures obtained after reversible, hot and cold rolling are presented in Figure 2 for the two alloys. The corresponding ODF's are published elsewhere ${ }^{8}$. They have been calculated without assuming a priori any sample symmetry.

\section{After reversible rolling (initial texture)}

Both materials are far from being isotropic since the maximum value of the ODF is equal to 33 (1200 alloy) and 66 (8979 alloy). The strongest component of texture is the cube $\{100\}<001>$ orientation in the case of the recrystallized material $(1200 \mathrm{Al})$ and the $S=\{123\}<634>$ (or more precisely one variant of this family of orientations) in the case of the 8979 alloy (in this case, the texture does not present a perfect symmetry).

\section{After cold and hot rolling}

As the rolling textures may be characterized adequately by complete descriptions of the $\alpha$ and $\beta$ fibres $(\alpha=\{110\}<u v w>$ and $\beta$ stretches from the copper orientation $\mathrm{Cu}$ $=\{112\}<111>$ to the brass component $B s=\{110\}<112>$ through $S$ and $D=$ 
$\{168\}<211>$ ), intensities along these fibres are presented in Figures 3 and 4 . Due to the lack of orthotropic symmetry, the $\alpha$ fibre is described by $\phi 1$ ranging from 0 to 180 , $\Phi=45$ and $\phi 2=0$. Concerning the $\beta$ fibre, only the maxima close to the 4 principal orientations listed above have been considered and plotted in Figure 4. Instead of one partial fibre (Cu-S-D-Bs), four partial fibres have to be investigated in our case (grouped into two fibres on the figures, named $\beta 1$ and $\beta 2$ ). In case of orthotropy, the two fibres would be identical and symmetrical with respect to the Bs orientation.
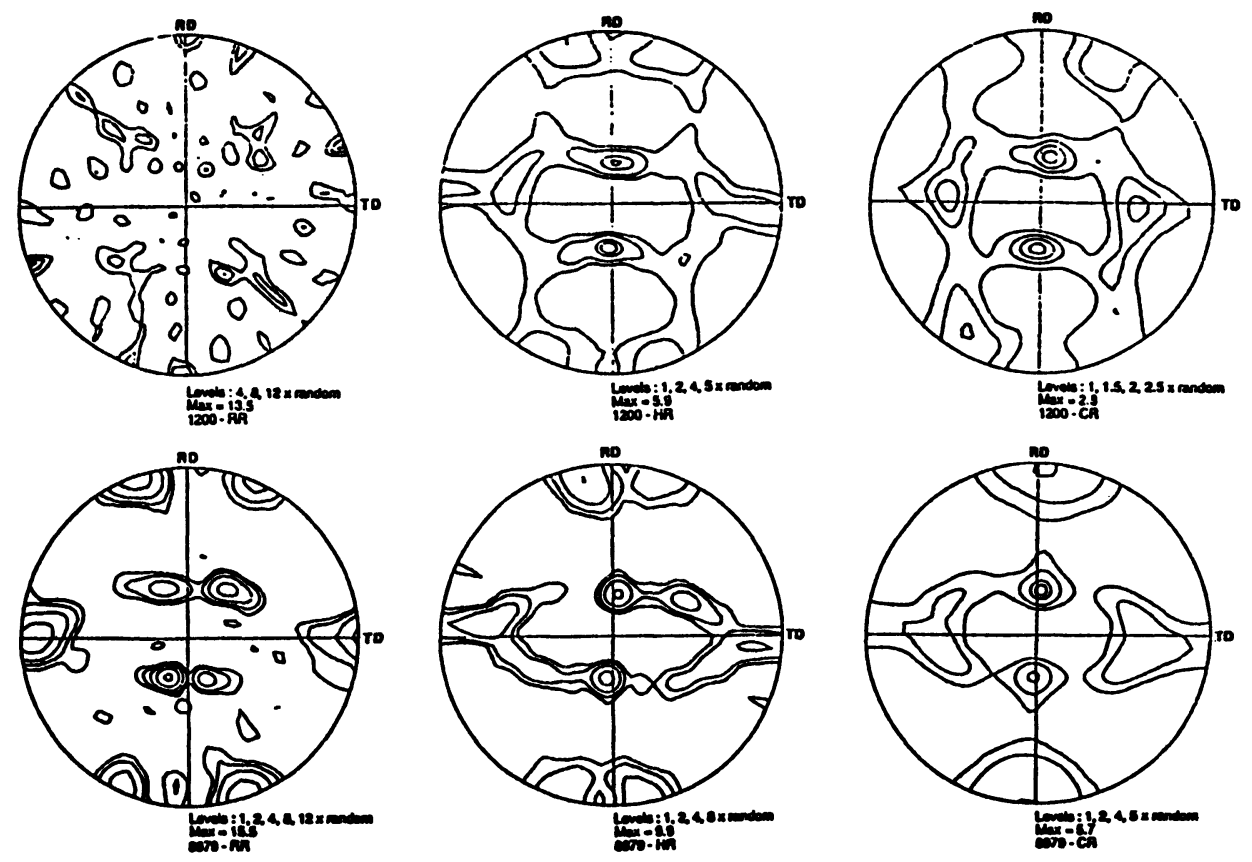

Figure 2 \{111\} pole figures measured for the 2 alloys after reversible (RR), hot (HR) and cold (CR) rolling.

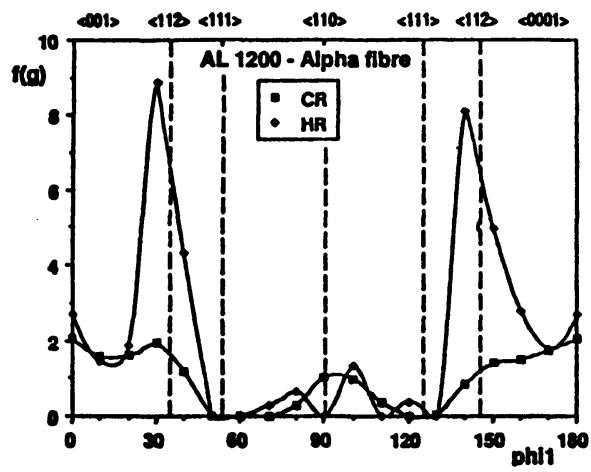

(a)

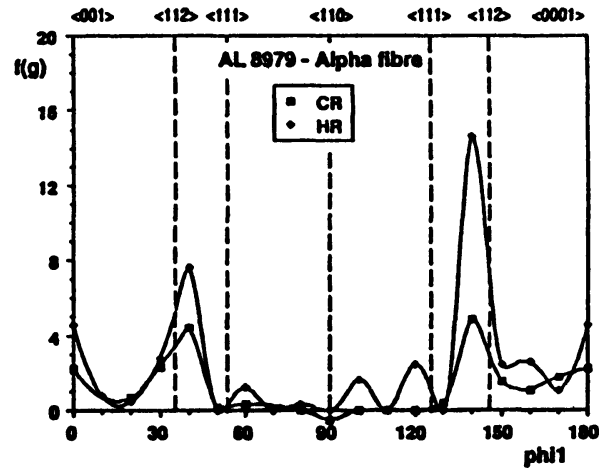

(b)

Figure 3 Intensities along the $\alpha$ fibre for a) the 1200 alloy and b) the 8979 alloy. 
The cold rolling results are consistent with published data concerning high SFE materials 2,4 : the orientations are spread along the $\beta$ fibre with a maximum on the $\mathrm{Cu}$ position. The texture developed in the recrystallized material is more or less orthotropic whereas the 8979 alloy has not recovered a complete symmetry after cold rolling. The maximum values of the ODF have strongly decreased (from 33 after reversible rolling to 6 after cold rolling for the 1200 alloy and from 62 to 15 for the 8979 alloy). After hot rolling, the textures of both materials are much stronger than after cold rolling (the maximum intensities are equal to 12.5 and 33 for the 1200 and 8979 alloys respectively). Furthermore, in both cases, the Bs intensity has increased.

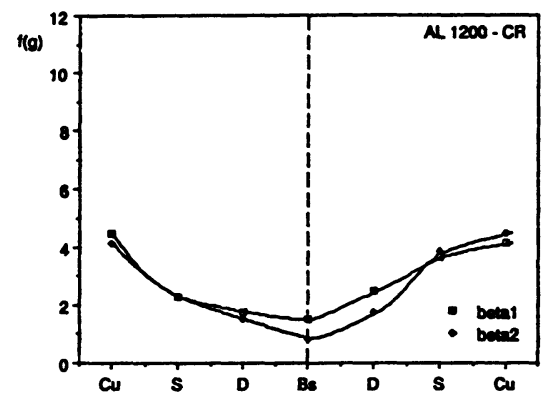

(a)
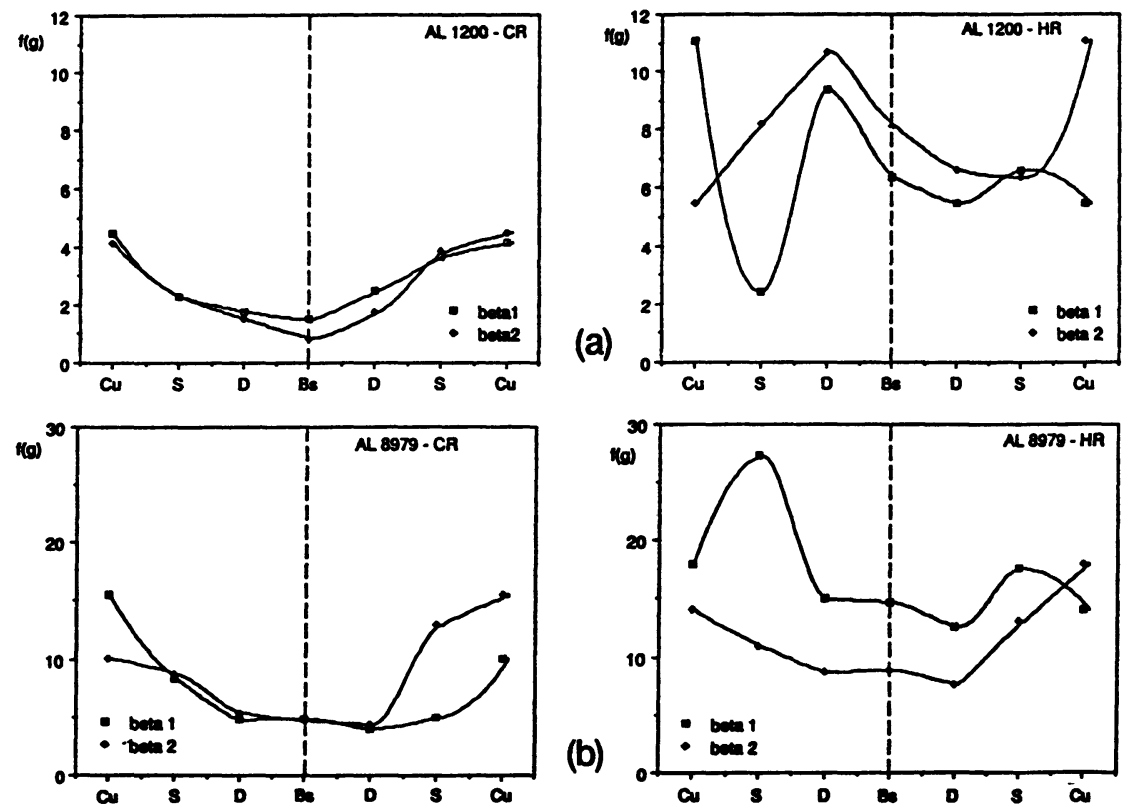

Figure 4 : Intensities along the $\beta$ fibre for a) the 1200 alloy and b) the 8979 alloy.

\section{DISCUSSION}

\section{Analysis of the results}

The textures of the two materials are very sharp after reversible rolling and they are also sharper after hot rolling than after cold rolling. This observation can be attributed to either a faster reorientation of the grains during hot rolling or to a retention of a strong initial texture. In the 1200 alloy, it is clear that that there is no retention of the initial texture since the $\mathrm{Cu}, \mathrm{Bs}$ and $\mathrm{S}$ components develop in both cases from the cube texture. In 8979 alloy, however, the results could be interpreted in terms of a partial retention of the $S$ and Bs orientations. However, the Cu component (absent from the initial texture) is stronger after hot than after cold rolling. Moreover, the intensity of the Bs component decreases during cold rolling and increases during hot rolling. It can thus be concluded that that there is an increasing rate of reorientation of grains with increasing temperature. The initial texture of the material has a strong influence on the intensity of the texture after deformation which is sharper when the material is initially 
non-recrystallized. However, the appearance of the Bs component during hot rolling is not due to the initial presence of a strong Goss component as was observed during cold rolling9. The development of a Bs component during hot rolling was also found by Hatherly et al. 3 in copper and Bull and LLyod ${ }^{10}$ in $\mathrm{Al}$ alloys and this result is in agreement with what is found during other deformation paths ${ }^{11-13}$.

The partial loss of orthotropy is also worthy of note. This can be attributed to an insufficient number of grains in the cross section of the material. If this could be argued for the reversible rolling case, this can not be true after hot rolling : the development of a very fine sub-structure (typical of dynamic recovery) in this case guarantees a good statistical quality of the texture measurements. Furthermore, it has been checked by repeated measurements that this observation was reproducible and that the true polycrystalline behaviour was in fact observed. It is believed that the limited number of passes during hot rolling does not allow a complete recovery of the rolling symmetry (which is not present after reversible rolling, also performed in a very limited number of passes). On the other hand, during cold rolling on the laboratory mill, the much greater number of passes tends to produce a better symmetry.

\section{Comparison with predicted textures}

Few attempts have been made up to now to introduce the effect of temperature in classical prediction models. Those that have been proposed fall into two main groups. Those that : i) change the deformation conditions, i.e. by assuming that the interaction conditions between one grain and its neighbours are different during hot and cold working ${ }^{11}$; ii) assume different deformation mechanisms within the grain (cross-slip, activation of new slip systems, climb of dislocation... ${ }^{11-13}$, viscoplastic glide ${ }^{14}$...).

The observed faster rate of reorientation with increasing temperature does not agree with the usual rate-sensitive power law which would predict exactly the opposite $^{14}$. However, this observation is in good agreement with the textures predicted by the self-consistent model when it takes into account the interaction between each grain and its nearest neighbours ${ }^{15}$. It is believed that preferred orientation relationships betweeen neighbouring grains (resulting from grain boudary energy minimization) have to be introduced in these models to account for high temperature behaviour.

Textures have been calculated under the assumption that all $\{111\},\{112\}$ and $\{110\}<110>$ systems can become active with the relaxed constraint model ${ }^{12}$ (Figure 5). The initial grain shapes and textures of the two materials have been taken into account : for the 1200 alloy, the grains were assumed to be equiaxed at the beginning of the calculation and to flatten during deformation, whereas for the 8979 alloy, the grain shape was assumed to be elongated from the beginning of the calculation. Furthermore, calculations were performed with collections of grains which reproduced the initial textures of the alloys (using the data from the vector method analysis). It is seen that a good agreement with experimental textures is found (a higher proportion of $S$ and Bs components can be found for the 8979 texture) and that the influence of the starting texture can not be neglected in the prediction of deformation textures. 

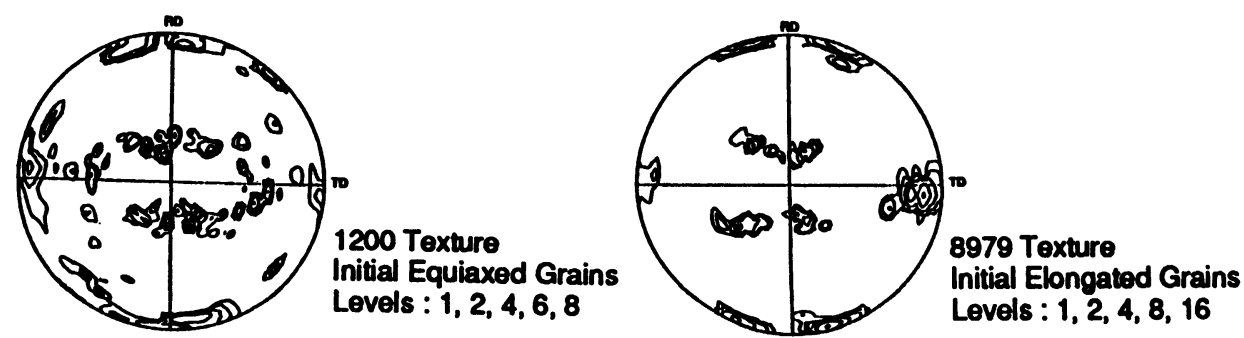

Figure 5 Predicted (111) rolling textures using the FC-RC model (see reference 12).

\section{CONCLUSION}

Textures of two Al alloys have been determined after cold and hot rolling in order to clarify the influence of temperature on these textures. Our principal observations are : (i) the texture strength increases with increasing temperature ; (ii) there is a significant increase of the Bs component with increasing temperature and (iii) the textures developed after hot rolling do not present a complete orthotropic symmetry. A good interpretation of the results needs : (i) a more complete experimental investigation, including single crystal experiments to identify the deformation mechanisms at various temepratures and (ii) the development of more appropriate models taking into account the mechanisms identified in the previous step. This is currently under investigation.

\section{ACKNOWLEDGEMENTS}

The authors gratefully acknowledge Dr. Ph. Lequeu from Pechiney (Research Center, Voreppe, France) for supplying all the experimental materials.

\section{REFERENCES}

1. Proc. Symp. on "Textures in Nonferrous Metals and Alloys", Detroit, MI, H.D. Merchant and J.G. Morris, eds, TMS-AIME (1984).

2. J. Hirsch and K. Lücke, Acta Metall., 36, 2863-2882 (1988).

3. M. Hatherly, A.S. Malin, C.M. Carmichael, F.J. Humphreys and J. Hirsch, Acta Metall., 34, 2247-2257 (1986).

4. P.A. Hollinshead and T. Sheppard, Metall. Trans., 20A, 1495-1507 (1989).

5. F. Montheillet, M. Cohen and J.J. Jonas, Acta Metall., 32, 2077-2089 (1984).

6. A. Vadon, J.J. Heizmann and C. Laruelle, in Proc. ICOTOM8, Santa Fé, J.S. Kallend and G. Gottstein eds., the Metallurgical Society, Inc., 87-92 (1987).

7. H.J. Bunge, "Texture Analysis in Materials Science", London, Butterworths, (1982).

8. O. Brun, Th. Chauveau and B. Bacroix, to appear in Mat. Sci. and Tech. (1990).

9. J. Hirsch, W. Mao and K. Lücke, in Proc. Al. Technology 86, London, 70 (1986). 10. M.J. Bull and DJ. Lloyd, in Proc. Conf. "Al-Li Alloys III", London, The Institute of Metals, $402-410$ (1985).

11. B. Bacroix, PhD thesis, McGill University, Montreal, CANADA (1986).

12. B. Bacroix and J.J. Jonas, Textures and Microstructures, 8 \& 9, 267-311 (1988).

13. B. Bacroix and J.J. Jonas, in Proc. ICOTOM8, Santa Fe, J.S. Kallend and G. Gottstein eds., the Metallurgical Society, Inc., $403-429$ (1987).

14. L.S. Toth, P. Gilormini and J.J. Jonas, Acta Metall., 36, 3077-3091 (1988).

15 A. Molinari, G.R. Canova and S. Ahzi, Acta Metall., 35, 2983-2994 (1987). 